\title{
Tight-and-Cheap Conic Relaxation for the Optimal Reactive Power Dispatch Problem
}

\author{
Christian Bingane, Student Member, IEEE, Miguel F. Anjos, Senior Member, IEEE and Sébastien Le Digabel
}

\begin{abstract}
The optimal reactive power dispatch (ORPD) problem is an alternating current optimal power flow (ACOPF) problem where discrete control devices for regulating the reactive power, such as shunt elements and tap changers, are considered. The ORPD problem is modelled as a mixed-integer nonlinear optimization problem and its complexity is increased compared to the ACOPF problem, which is highly nonconvex and generally hard to solve. Recently, convex relaxations of the ACOPF problem have attracted a significant interest since they can lead to global optimality. We propose a tight conic relaxation of the ORPD problem and show that a round-off technique applied with this relaxation leads to near-global optimal solutions with very small guaranteed optimality gaps, unlike with the nonconvex continuous relaxation. We report computational results on selected MATPOWER test cases with up to 3375 buses.
\end{abstract}

Index Terms-Conic optimization, discrete variables, optimal power flow, power systems, semidefinite programming.

\section{NOMENCLATURE}

\section{A. Notations}

$\mathbb{R} / \mathbb{C}$

$\mathbb{H}^{n}$

$\mathrm{j}$

$a / \mathrm{a}$

a/a

A/A

Set of real/complex numbers,

Set of $n \times n$ Hermitian matrices,

Imaginary unit,

Real/complex number,

Real/complex vector,

Real/complex matrix.

\section{B. Operators}

$\operatorname{Re}(\cdot) / \operatorname{Im}(\cdot)$

$(\cdot)^{*}$

$|\cdot|$

$\angle(\cdot)$

$(\cdot)^{H}$

Real/imaginary part operator,

Conjugate operator,

Magnitude or cardinality set operator,

Phase operator,

Conjugate transpose operator.

\section{Input data}

$\mathscr{P}=(\mathcal{N}, \mathcal{L})$

$\mathcal{N}$

$\mathcal{U} \subseteq \mathcal{N}$

Power network,

Set of buses,

$\mathcal{G}=\bigcup_{k \in \mathcal{N}} \mathcal{G}_{k} \quad \begin{aligned} & \text { connected, } \\ & \text { Set of generators, }\end{aligned}$

The second author is with the School of Mathematics, University of Edinburgh, Edinburgh EH9 3FD, Scotland, UK.

All authors are with the Department of Mathematics and Industrial Engineering, Polytechnique Montreal, Montreal, Quebec, Canada H3C 3A7; and the GERAD research center, Montreal, Quebec, Canada H3T 2A7.

E-mails: christian.bingane@polymtl.ca, anjos@stanfordalumni.org, sebastien.le-digabel@polymtl.ca.

This research was supported by the NSERC-Hydro-Quebec-Schneider Electric Industrial Research Chair.
$\mathcal{G}_{k}$

$\mathcal{L}$

$\mathcal{T} \subseteq \mathcal{L}$

$p_{D k} / q_{D k}$

$g_{k}^{\prime} / b_{k}^{\prime}$

$c_{g 2}, c_{g 1}, c_{g 0}$

$\mathrm{y}_{\ell}^{-1}=r_{\ell}+\mathrm{j} x_{\ell}$

$b_{\ell}^{\prime}$

D. Variables

$p_{G g} / q_{G g}$

$\mathrm{v}_{k}$

$u_{k}$

$p_{f \ell} / q_{f \ell}$

$p_{t \ell} / q_{t \ell}$

$t_{\ell}$

Active/reactive power generation by generator $g$, Complex (phasor) voltage at bus $k$, Shunt variable corresponding to the shunt element connected at bus $k$,

Active/reactive power flow injected along branch $\ell$ by its from end,

Active/reactive power flow injected along branch $\ell$ by its to end,

Turns ratio of tap changer $\ell$.

\section{INTRODUCTION}

$\mathbf{T}$ HE optimal power flow (OPF) problem, first formulated in [1], consists in finding a network operating point that optimizes an objective function subject to power flow equations and other operational constraints [2]-[5]. The continuous version with $\mathrm{AC}$ power flow equations, also called $\mathrm{AC}$ optimal power flow (ACOPF) problem, is nonconvex and NPhard [6], [7]. The optimal reactive power dispatch (ORPD) problem, also known as Volt/VAR optimization problem, can be seen as an ACOPF problem with discrete control devices for regulating the reactive power such as shunt elements and tap changers [8], [9]. Because of the presence of discrete variables, the ORPD problem is generally more difficult than the ACOPF.

A mixed-integer nonlinear program (MINLP) is an optimization problem which involves both continuous and integer variables and whose objective function and feasible set are described by nonlinear functions [10]. A MINLP is said to be convex if its continuous relaxation, i.e. the problem obtained by dropping the integrality constraints, is a convex optimization problem; otherwise, it is said to be nonconvex. MINLPs inherit difficulties from nonlinear programs (NLPs) and mixed-integer linear programs (MILPs) since they are a generalization of both classes. In fact, there exist simple cases of nonconvex MINLPs which are not only NP-hard, but even undecidable. See more details in [11], [12]. 
Recently, convex relaxations of the ACOPF problem, in particular second-order cone programming (SOCP) [13], semidefinite programming (SDP) [14], and quadratic convex (QC) [15] relaxations, have attracted a significant interest for several reasons. First, they can lead to global optimality; second, because they are relaxations, they provide a bound on the global optimal value of the ACOPF problem; and third, if one of these relaxations is infeasible, then the ACOPF problem is infeasible. Mixed-integer QC relaxations were proposed in [16] and mixed-integer SOCP relaxations in [17]-[19] for MINLPs in power systems. However, [5] emphasized that convex relaxations of the OPF problem are aimed at complementing nonconvex (local) solvers with the valuable information about the quality of the solution obtained, rather than at replacing them.

Moreover, several applications of OPF are multi-period problems by nature due to factors such as changing market prices, ramping limits of generation units, and demand behavior [20]. Extending a convex relaxation from a singleperiod OPF problem to a multi-period one may jeopardize its exactness. This is discussed in [21] where a tight convex relaxation for the multi-period case is proposed.

On the other hand, some heuristic techniques discussed in [22] have been proposed for handling discrete variables in the OPF problem. One approach works as follows: first, solve the continuous relaxation of the OPF problem, i.e. treat the discrete variables as continuous; second, round-off solutions corresponding to discrete variables to their nearest discrete values; and third, fix discrete variables to these values and then solve the corresponding OPF subproblem. This approach is called the round-off technique and remains the simplest to deal with discrete variables in the ORPD problem, although it may lead to poor suboptimal solutions or infeasible ones. Some deficiences were already pointed out in [23]: for instance, since the OPF problem is in general highly nonconvex, solving its continuous relaxation with a local optimizer in the first step may lead to a poor local solution which, after round-off in the second step, may lead to a very poor solution in the third step. More discussion about the round-off strategy can be found in [5], [9].

The main contribution of this paper is to show that a roundoff technique used with a tight convex continuous relaxation may lead to near-global optimal solutions of the ORPD problem. This contribution is in two parts. First, we propose SDP-based relaxations of the ORPD problem with a new tight convex model of tap changer; second, a modified round-off technique where, in the first step, we solve a SDP-based relaxation instead of the nonconvex continuous relaxation of the ORPD problem. More details about semidefinite optimization can be found in [24]. Computational results show that the SDP-based relaxations of the ORPD problem are tight, and furthermore that applying the round-off technique with these relaxations leads to near-global solutions, even for large-scale instances. To the best of our knowledge, it is the first time that extensive computations with this approach are carried out for the ORPD problem with large-scale meshed networks.

The remainder of this paper is organized as follows. In Section II, we state the mathematical model of the ORPD problem.
In Section III, we describe two SDP-based relaxations of the ORPD problem: a simple one already proposed in [25] and a new tighter one. In Section IV, we present our round-off technique, and we report computational results in Section V. Section VI concludes the paper.

\section{ORPD: FORMULATION}

Consider a typical power network $\mathscr{P}=(\mathcal{N}, \mathcal{L})$, where $\mathcal{N}=$ $\{1,2, \ldots, n\}$ and $\mathcal{L} \subseteq \mathcal{N} \times \mathcal{N}$ denote respectively the set of buses and the set of branches (transmission lines and tap changers). We denote by $\mathcal{U} \subseteq \mathcal{N}$ the set of buses $k$ where a shunt element is connected, and $\mathcal{T} \subseteq \mathcal{L}$ the set of branches with tap changers. Each branch $\ell \in \mathcal{L}$ has a from end $k$ (on the tap side) and a to end $m$ as modeled in [26]. We denote $\ell=(k, m)$. The ORPD problem is given as:

$$
\min f\left(\boldsymbol{u}, \boldsymbol{t}, \boldsymbol{p}_{G}, \boldsymbol{q}_{G}, \boldsymbol{p}_{f}, \boldsymbol{q}_{f}, \boldsymbol{p}_{t}, \boldsymbol{q}_{t}, \mathbf{v}\right)
$$

over variables $\boldsymbol{u} \in\{0,1\}^{|\mathcal{U}|}, \boldsymbol{t} \in \mathbb{R}^{|\mathcal{T}|}, \boldsymbol{p}_{G}, \boldsymbol{q}_{G} \in \mathbb{R}^{|\mathcal{G}|}$, $\boldsymbol{p}_{f}, \boldsymbol{q}_{f}, \boldsymbol{p}_{t}, \boldsymbol{q}_{t} \in \mathbb{R}^{|\mathcal{L}|}$, and $\mathbf{v} \in \mathbb{C}^{|\mathcal{N}|}$, subject to

- Power balance equations:

$$
\begin{aligned}
& \sum_{g \in \mathcal{G}_{k}} p_{G g}-p_{D k}-g_{k}^{\prime} u_{k}\left|\mathrm{v}_{k}\right|^{2} \\
& =\sum_{\ell=(k, m) \in \mathcal{L}} p_{f \ell}+\sum_{\ell=(m, k) \in \mathcal{L}} p_{t \ell} \forall k \in \mathcal{U}, \\
& \sum_{g \in \mathcal{G}_{k}} q_{G g}-q_{D k}+b_{k}^{\prime} u_{k}\left|\mathrm{v}_{k}\right|^{2} \\
& =\sum_{\ell=(k, m) \in \mathcal{L}} q_{f \ell}+\sum_{\ell=(m, k) \in \mathcal{L}} q_{t \ell} \forall k \in \mathcal{U}, \\
& \sum_{g \in \mathcal{G}_{k}} p_{G g}-p_{D k} \\
& =\sum_{\ell=(k, m) \in \mathcal{L}} p_{f \ell}+\sum_{\ell=(m, k) \in \mathcal{L}} p_{t \ell} \forall k \in \mathcal{N} \backslash \mathcal{U}, \\
& \sum_{g \in \mathcal{G}_{k}} q_{G g}-q_{D k} \sum_{\ell=(k, m) \in \mathcal{L}} q_{f \ell}+\sum_{\ell=(m, k) \in \mathcal{L}} q_{t \ell} \forall k \in \mathcal{N} \backslash \mathcal{U},
\end{aligned}
$$

- Branch flow equations:

$$
\begin{aligned}
& \frac{\mathrm{v}_{k}}{t_{\ell}}\left[\left(\mathrm{j} \frac{b_{\ell}^{\prime}}{2}+\mathrm{y}_{\ell}\right) \frac{\mathrm{v}_{k}}{t_{\ell}}-\mathrm{y}_{\ell} \mathrm{v}_{m}\right]^{*} \\
& =p_{f \ell}+\mathrm{j} q_{f \ell} \forall \ell=(k, m) \in \mathcal{T}, \\
& \mathrm{v}_{m}\left[-\mathrm{y}_{\ell} \frac{\mathrm{v}_{k}}{t_{\ell}}+\left(\mathrm{j} \frac{b_{\ell}^{\prime}}{2}+\mathrm{y}_{\ell}\right) \mathrm{v}_{m}\right]^{*} \\
& =p_{t \ell}+\mathrm{j} q_{t \ell} \forall \ell=(k, m) \in \mathcal{T}, \\
& \mathrm{v}_{k}\left[\left(\mathrm{j} \frac{b_{\ell}^{\prime}}{2}+\mathrm{y}_{\ell}\right) \mathrm{v}_{k}-\mathrm{y}_{\ell} \mathrm{v}_{m}\right]^{*} \\
& =p_{f \ell}+\mathrm{j} q_{f \ell} \forall \ell=(k, m) \in \mathcal{L} \backslash \mathcal{T}, \\
& \mathrm{v}_{m}\left[-\mathrm{y}_{\ell} \mathrm{v}_{k}+\left(\mathrm{j} \frac{b_{\ell}^{\prime}}{2}+\mathrm{y}_{\ell}\right) \mathrm{v}_{m}\right]^{*} \\
& =p_{t \ell}+\mathrm{j} q_{t \ell} \forall \ell=(k, m) \in \mathcal{L} \backslash \mathcal{T},
\end{aligned}
$$


- Generator power capacities:

$$
\underline{p}_{G g} \leq p_{G g} \leq \bar{p}_{G g}, \underline{q}_{G g} \leq q_{G g} \leq \bar{q}_{G g} \forall g \in \mathcal{G},
$$

- Line thermal limits:

$$
\left|p_{f \ell}+\mathrm{j} q_{f \ell}\right| \leq \bar{s}_{\ell},\left|p_{t \ell}+\mathrm{j} q_{t \ell}\right| \leq \bar{s}_{\ell} \forall \ell \in \mathcal{L},
$$

- Voltage magnitude limits:

$$
\underline{v}_{k} \leq\left|\mathrm{v}_{k}\right| \leq \bar{v}_{k} \forall k \in \mathcal{N},
$$

- Reference bus constraint:

$$
\angle \mathrm{v}_{1}=0,
$$

- Shunt variable:

$$
u_{k} \in\{0,1\} \forall k \in \mathcal{U},
$$

- Tap ratio constraint:

$$
t_{\ell} \in\left\{\underline{t}_{\ell}, \ldots, \bar{t}_{\ell}\right\} \forall \ell \in \mathcal{T} .
$$

Constraints (1b)-(1i) derive from Kirchhoff's laws and represent power flow equations in the network. We assume $\underline{v}_{k}>0$ for all $k \in \mathcal{N}$ in (11) and $\underline{t}_{\ell}>0$ for all $\ell \in \mathcal{T}$ in (1o). Constraint $(1 \mathrm{~m})$ specifies bus $k=1$ as the reference bus. In (1n), we assume that the shunt connected to a bus $k \in \mathcal{U}$ has an on/off switch. For all $\ell \in \mathcal{T}$, the tap ratio $t_{\ell}$ in (1o) is a discrete variable that typically takes on $2 \bar{\eta}+1$ values $\left\{\hat{t}_{-\bar{\eta}}, \ldots, \hat{t}_{0}, \ldots, \hat{t}_{\bar{\eta}}\right\}$, uniformly distributed around $\hat{t}_{0}$. In this paper, we assume without loss of generality that $\hat{t}_{\ell}$ varies between 0.9 and $1.1 \mathrm{pu}$ with steps of $0.0125 \mathrm{pu}$ (17 different settings) for all $\ell \in \mathcal{T}$.

The objective function $f\left(\boldsymbol{u}, \boldsymbol{t}, \boldsymbol{p}_{G}, \boldsymbol{q}_{G}, \boldsymbol{p}_{f}, \boldsymbol{q}_{f}, \boldsymbol{p}_{t}, \boldsymbol{q}_{t}, \mathbf{v}\right)$ in (1a) may represent any objective function related to the ORPD problem: power loss, voltage deviation, number of control actions, generation cost, etc. Some of them are considered in [27]. We note that power loss is a widely used objective function for the ORPD problem. However, according to [2], just minimizing loss is inconsistent with economic principles and may result in suboptimal dispatch while minimizing cost would be the correct objective function for economically dispatching resources and would inherently meet the objective of minimizing loss. Recently, [9] considered minimizing generation cost instead of power loss in their formulation of the ORPD problem. In this work, we consider both cost minimization and loss minimization in our computational results. We also assume that (1a) is a convex function, and then relaxations proposed in this paper remain valid for any convex objective function related to the ORPD problem.

\section{ORPD: CONVEXIFICATION}

In this section, we describe two semidefinite relaxations of the ORPD problem: a simple one (SDR1) already proposed in [25] and a new tighter one (SDR2). SDR2 is obtained by combining SDR1 with a new tight convex model of tap changer and we show that SDR2 is then stronger than SDR1. Since both SDR1 and SDR2 can be expensive to solve for large-scale instances, we derive from them two relaxations that are cheaper to solve: TCR1 and TCR2 respectively.

\section{A. Semidefinite relaxation 1 (SDR1)}

Let

$$
\begin{array}{rlrl}
\mathrm{V} & :=\mathbf{v v}^{H}, & \\
\mathrm{~W}_{\{\ell\}}: & =\left[\begin{array}{lll}
\mathrm{V}_{k k} & \mathrm{~W}_{k \ell} & \mathrm{V}_{k m} \\
\mathrm{~W}_{k \ell}^{*} & \mathrm{~W}_{\ell \ell} & \mathrm{W}_{\ell m} \\
\mathrm{~V}_{k m}^{*} & \mathrm{~W}_{\ell m}^{*} & \mathrm{~V}_{m m}
\end{array}\right] \\
& :=\left[\begin{array}{c}
\mathrm{v}_{k} \\
\mathrm{w}_{\ell} \\
\mathrm{v}_{m}
\end{array}\right]\left[\begin{array}{c}
\mathrm{v}_{k} \\
\mathrm{w}_{\ell} \\
\mathrm{v}_{m}
\end{array}\right] & \forall \ell=(k, m) \in \mathcal{T}, \\
\mathrm{w}_{\ell} & :=\frac{\mathrm{v}_{k}}{t_{\ell}} & \forall \ell=(k, m) \in \mathcal{T} .
\end{array}
$$

The ORPD problem (1) can be reformulated as follows

minimize (1a)

subject to (1d), (1e), (1j), (1k), (1m), (1n), (1o),

$$
\begin{aligned}
& \sum_{g \in \mathcal{G}_{k}} p_{G g}-p_{D k}-g_{k}^{\prime} u_{k} \mathrm{~V}_{k k} \\
& =\sum_{\ell=(k, m) \in \mathcal{L}} p_{f \ell}+\sum_{\ell=(m, k) \in \mathcal{L}} p_{t \ell} \forall k \in \mathcal{U}, \\
& \sum_{g \in \mathcal{G}_{k}} q_{G g}-q_{D k}+b_{k}^{\prime} u_{k} \mathrm{~V}_{k k} \\
& =\sum_{\ell=(k, m) \in \mathcal{L}} q_{f \ell}+\sum_{\ell=(m, k) \in \mathcal{L}} q_{t \ell} \forall k \in \mathcal{U}, \\
& \left(-\mathrm{j} \frac{b_{\ell}^{\prime}}{2}+\mathrm{y}_{\ell}^{*}\right) \mathrm{W}_{\ell \ell}-\mathrm{y}_{\ell}^{*} \mathrm{~W}_{\ell m} \\
& =p_{f \ell}+\mathrm{j} q_{f \ell} \forall \ell=(k, m) \in \mathcal{T} \\
& -\mathrm{y}_{\ell}^{*} \mathrm{~W}_{\ell m}^{*}+\left(-\mathrm{j} \frac{b_{\ell}^{\prime}}{2}+\mathrm{y}_{\ell}^{*}\right) \mathrm{V}_{m m} \\
& =p_{t \ell}+\mathrm{j} q_{t \ell} \forall \ell=(k, m) \in \mathcal{T}, \\
& \left(-\mathrm{j} \frac{b_{\ell}^{\prime}}{2}+\mathrm{y}_{\ell}^{*}\right) \mathrm{V}_{k k}-\mathrm{y}_{\ell}^{*} \mathrm{~V}_{k m} \\
& =p_{f \ell}+\mathrm{j} q_{f \ell} \forall \ell=(k, m) \in \mathcal{L} \backslash \mathcal{T}, \\
& -\mathrm{y}_{\ell}^{*} \mathrm{~V}_{k m}^{*}+\left(-\mathrm{j} \frac{b_{\ell}^{\prime}}{2}+\mathrm{y}_{\ell}^{*}\right) \mathrm{V}_{m m} \\
& =p_{t \ell}+\mathrm{j} q_{t \ell} \forall \ell=(k, m) \in \mathcal{L} \backslash \mathcal{T}, \\
& \underline{v}_{k}^{2} \leq \mathrm{V}_{k k} \leq \bar{v}_{k}^{2} \forall k \in \mathcal{N} .
\end{aligned}
$$

If we define $\xi_{k}:=u_{k} \mathrm{~V}_{k k} \in\left\{0, \mathrm{~V}_{k k}\right\}$ for all $k \in \mathcal{U}$, a linear formulation of (3a)-(3b) is given as

$$
\begin{aligned}
& \sum_{g \in \mathcal{G}_{k}} p_{G g}-p_{D k}-g_{k}^{\prime} \xi_{k} \\
& =\sum_{\ell=(k, m) \in \mathcal{L}} p_{f \ell}+\sum_{\ell=(m, k) \in \mathcal{L}} p_{t \ell} \forall k \in \mathcal{U}, \\
& \sum_{g \in \mathcal{G}_{k}} q_{G g}-q_{D k}+b_{k}^{\prime} \xi_{k} \\
& =\sum_{\ell=(k, m) \in \mathcal{L}} q_{f \ell}+\sum_{\ell=(m, k) \in \mathcal{L}} q_{t \ell} \forall k \in \mathcal{U}, \\
& 0 \leq \xi_{k} \leq \mathrm{V}_{k k} \forall k \in \mathcal{U} \text {. }
\end{aligned}
$$


From (2b) and (2c), we have

$$
\begin{array}{ll}
\mathrm{W}_{k \ell}=\frac{\mathrm{V}_{k k}}{t_{\ell}} & \forall \ell=(k, m) \in \mathcal{T}, \\
\mathrm{W}_{\ell \ell}=\frac{\mathrm{V}_{k k}}{t_{\ell}^{2}} & \forall \ell=(k, m) \in \mathcal{T},
\end{array}
$$

which implies

$$
\begin{array}{ll}
\frac{\mathrm{V}_{k k}}{\bar{t}_{\ell}} \leq \mathrm{W}_{k \ell} \leq \frac{\mathrm{V}_{k k}}{\underline{t}_{\ell}} & \forall \ell=(k, m) \in \mathcal{T}, \\
\frac{\mathrm{V}_{k k}}{\bar{t}_{\ell}^{2}} \leq \mathrm{W}_{\ell \ell} \leq \frac{\mathrm{V}_{k k}}{\underline{t}_{\ell}^{2}} & \forall \ell=(k, m) \in \mathcal{T},
\end{array}
$$

since $0<\underline{t}_{\ell} \leq t_{\ell} \leq \bar{t}_{\ell}$ for all $\ell \in \mathcal{T}$. Finally, we can show that $\mathrm{V}$ in (2a) and, for all $\ell \in \mathcal{T}, \mathrm{W}_{\{\ell\}}$ in (2b) are rank-one positive semidefinite matrices. The semidefinite relaxation 1 (SDR1) in Model 1 is obtained by dropping the rank constraints. For all $\ell=(k, m) \in \mathcal{T}$, since $\mathrm{W}_{\{\ell\}} \succeq 0$ implies $\mathrm{W}_{k \ell}^{2} \leq \mathrm{V}_{k k} \mathrm{~W}_{\ell \ell}$, (6) can be rewritten as

$$
\begin{array}{ll}
\mathrm{W}_{k \ell} \geq \frac{\mathrm{V}_{k k}}{\bar{t}_{\ell}} & \forall \ell=(k, m) \in \mathcal{T}, \\
\mathrm{W}_{\ell \ell} \leq \frac{\mathrm{V}_{k k}}{\underline{t}_{\ell}^{2}} & \forall \ell=(k, m) \in \mathcal{T} .
\end{array}
$$

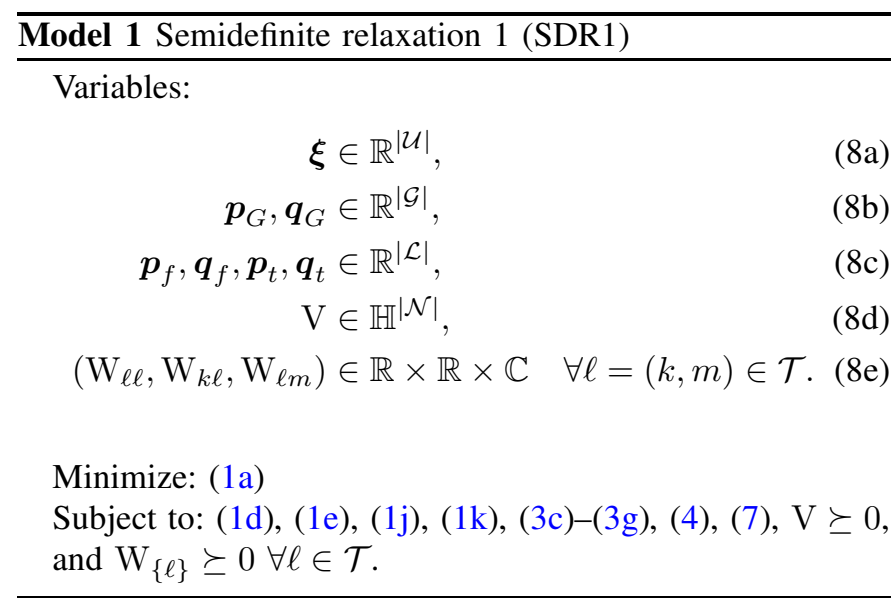

We point out that the variables $u_{k}$ and $t_{\ell}$ are eliminated in Model 1 for all $k \in \mathcal{U}$ and for all $\ell \in \mathcal{T}$. This approach was already proposed in [25] and was recently used in [28]. Once the solution of Model 1 is obtained, the optimal value $\hat{u}_{k}$ of the shunt element connected to a bus $k \in \mathcal{U}$ and the optimal tap ratio $\hat{t}_{\ell}$ of a transformer $\ell=(k, m) \in \mathcal{T}$ can be determined respectively as follows:

$$
\begin{gathered}
\hat{u}_{k}=\frac{\xi_{k}}{\mathrm{~V}_{k k}} \in[0,1], \\
\hat{t}_{\ell}=\sqrt{\frac{\mathrm{V}_{k k}}{\mathrm{~W}_{\ell \ell}}} \in\left[\underline{t}_{\ell}, \bar{t}_{\ell}\right] .
\end{gathered}
$$

If the optimal solutions $\hat{\mathrm{V}}$ and $\hat{\mathrm{W}}_{\{\ell\}}$ for all $\ell \in \mathcal{T}$ are rankone matrices, $\hat{u}_{k} \in\{0,1\}$ for all $k \in \mathcal{U}$, and $\hat{t}_{\ell} \in\left\{\underline{t}_{\ell}, \ldots, \bar{t}_{\ell}\right\}$ for all $\ell \in \mathcal{T}$, then there exists a complex vector $\hat{\mathbf{v}}$ that is a global optimal solution of (1). We say that the relaxation SDR1 is exact.

We also consider a cheaper relaxation, called "tight-and- cheap relaxation 1", given in Model 2 and obtained as follows. We replace $\mathrm{V} \succeq 0$ and $\mathrm{W}_{\{\ell\}} \succeq 0$ for all $\ell \in \mathcal{T}$ in Model 1 by

$$
\begin{array}{rlr}
{\left[\begin{array}{ccc}
1 & \mathrm{v}_{k}^{*} & \mathrm{v}_{m}^{*} \\
\mathrm{v}_{k} & \mathrm{~V}_{k k} & \mathrm{~V}_{k m} \\
\mathrm{v}_{m} & \mathrm{~V}_{k m}^{*} & \mathrm{~V}_{m m}
\end{array}\right]} & \succeq 0 \quad \forall \ell=(k, m) \in \mathcal{L} \backslash \mathcal{T}, \\
{\left[\begin{array}{cc}
1 & \mathbf{w}_{\{\ell\}}^{H} \\
\mathbf{w}_{\{\ell\}} & \mathrm{W}_{\{\ell\}}
\end{array}\right] \succeq 0 \quad \forall \ell=(k, m) \in \mathcal{T},}
\end{array}
$$

where $\mathbf{w}_{\{\ell\}}^{H}=\left(\mathrm{v}_{k}^{*}, \mathrm{w}_{\ell}^{*}, \mathrm{v}_{m}^{*}\right)$ for all $\ell=(k, m) \in \mathcal{T}$, and we add the following constraints

$$
\begin{aligned}
& \operatorname{Re}\left(\mathrm{v}_{1}\right) \geq \frac{\mathrm{V}_{11}+\underline{v}_{1} \bar{v}_{1}}{\underline{v}_{1}+\bar{v}_{1}}, \\
& \operatorname{Im}\left(\mathrm{v}_{1}\right)=0,
\end{aligned}
$$

corresponding to the reference bus $k=1$.

\begin{tabular}{l}
\hline Model 2 Tight-and-cheap relaxation 1 (TCR1) \\
\hline Variables: (8), $\mathbf{v} \in \mathbb{C}^{|\mathcal{N}|}, \mathbf{w} \in \mathbb{C}^{|\mathcal{T}|}$. \\
Minimize: (1a) \\
Subject to: (1d), (1e), (1j), (1k), (3c)-(3g), (4), (6), (10). \\
\hline
\end{tabular}

The tight-and-cheap relaxation (TCR) was first proposed in [29] for the ACOPF problem. It was shown in [29] that TCR is stronger than the standard SOCP relaxation and nearly as tight as the standard SDP relaxation. Moreover, computation experiments on standard test cases with up to 6515 buses showed that solving TCR for large-scale instances is much less expensive than solving the chordal relaxation, a SDP relaxation technique that exploits the sparsity of power networks.

\section{B. Semidefinite relaxation 2 (SDR2)}

For all $\ell=(k, m) \in \mathcal{T}$, variables $\mathrm{W}_{k \ell}$ and $\mathrm{W}_{\ell \ell}$ in (5) are respectively described by constraints of the form $z_{n}=x / y^{n}$, $n=1,2$. Consider the set

$$
\begin{array}{r}
\mathcal{S}_{1}=\left\{\left(x, y, z_{1}, z_{2}\right) \in \mathbb{R}^{4}: \underline{x} \leq x \leq \bar{x}, \underline{y} \leq y \leq \bar{y},\right. \\
\left.z_{1}=x / y, z_{2}=x / y^{2}\right\},
\end{array}
$$

where $0<\underline{x}<\bar{x}$ and $0<\underline{y}<\bar{y}$. We can show that $\mathcal{S}_{1}$ is equivalent to

$$
\mathcal{S}_{2}=\left\{\left(x, z_{1}, z_{2}\right) \in \mathbb{R}^{3}: z_{2}=z_{1}^{2} / x,\left(x, z_{1}\right) \in \Omega\right\},
$$

where $\Omega=\left\{\left(x, z_{1}\right) \in \mathbb{R}^{2}: \underline{x} \leq x \leq \bar{x}, x / \bar{y} \leq z_{1} \leq x / \underline{y}\right\}$ is the convex quadrilateral with vertices $(\underline{x}, \underline{x} / \bar{y}),(\bar{x}, \bar{x} / \bar{y})$, $(\bar{x}, \bar{x} / \underline{y})$, and $(\underline{x}, \underline{x} / \underline{y})$.

In Section III-A, the proposed convex set that contains $\mathcal{S}_{2}$ is

$$
\begin{aligned}
\mathcal{S}_{3}=\left\{\left(x, z_{1}, z_{2}\right) \in \mathbb{R}^{3}:\right. & \underline{x} \leq x \leq \bar{x}, \\
& \left.z_{2} \geq z_{1}^{2} / x, z_{1} \geq x / \bar{y}, z_{2} \leq x / \underline{y}^{2}\right\} .
\end{aligned}
$$

In this section, we propose a tighter convex set containing $\mathcal{S}_{2}$.

Lemma 1. Let $f\left(x, z_{1}\right)=z_{1}^{2} / x$ defined over $\mathbb{R}_{+} \times \mathbb{R}$. Then $f$ is convex.

Proof: For all $\left(x, z_{1}\right) \in \mathbb{R}_{+} \times \mathbb{R}$,

$$
\nabla^{2} f\left(x, z_{1}\right)=\frac{2}{x^{3}}\left[\begin{array}{cc}
z_{1}^{2} & -x z_{1} \\
-x z_{1} & x^{2}
\end{array}\right]=\frac{2}{x^{3}}\left[\begin{array}{c}
z_{1} \\
-x
\end{array}\right]\left[\begin{array}{c}
z_{1} \\
-x
\end{array}\right]^{T} \succeq 0 .
$$


Then $f$ is convex.

Lemma 2. Let $\left(x, z_{1}\right) \in \Omega$. If $z_{2}=z_{1}^{2} / x$, then $x+\underline{y} \bar{y} z_{2} \leq$ $(\underline{y}+\bar{y}) z_{1}$.

Proof: Let $\left(x, z_{1}\right) \in \Omega$ and $z_{2}=z_{1}^{2} / x$. We have $x / \bar{y} \leq$ $z_{1} \leq x / \underline{y}$ with $0<\underline{y}<\bar{y}$. Therefore, $\left(x-\underline{y} z_{1}\right)\left(x-\bar{y} z_{1}\right) \leq 0$. On the other hand,

$$
\begin{aligned}
\left(x-\underline{y} z_{1}\right)\left(x-\bar{y} z_{1}\right) & =x^{2}-(\underline{y}+\bar{y}) x z_{1}+\underline{y} \bar{y} z_{1}^{2} \\
& =x\left[x-(\underline{y}+\bar{y}) z_{1}+\underline{y} \bar{y} z_{2}\right] \leq 0 .
\end{aligned}
$$

Since $\underline{x}>0$, it follows that $x-(\underline{y}+\bar{y}) z_{1}+\underline{y} \bar{y} z_{2} \leq 0$.

Proposition 1. Let $f\left(x, z_{1}\right)=z_{1}^{2} / x$ defined over $\Omega$. The concave envelope or the tightest concave overestimator of $f$ on $\Omega$ is $h\left(x, z_{1}\right)=\frac{1}{\underline{y} \bar{y}}\left[(\underline{y}+\bar{y}) z_{1}-x\right]$.

Proof: The function $h$ is affine and, from Lemma 2, overestimates $f$ on $\Omega$. Then $h$ is a concave overestimator of $f$ on $\Omega$. We note that $h\left(x, z_{1}\right)=f\left(x, z_{1}\right)$ for all $\left(x, z_{1}\right) \in \Omega$ such that $z_{1}=x / \bar{y}$ or $z_{1}=x / \underline{y}$.

Suppose that there exists a concave overestimator $\tilde{h}$ of $f$ on $\Omega$ such that $f\left(x, z_{1}\right) \leq \tilde{h}\left(x, z_{1}\right) \leq h\left(x, z_{1}\right)$ for all $\left(x, z_{1}\right) \in$ $\Omega$ and $\tilde{h}\left(\tilde{x}, \tilde{z}_{1}\right)<h\left(\tilde{x}, \tilde{z}_{1}\right)$ for some $\left(\tilde{x}, \tilde{z}_{1}\right) \in \Omega$. Since $\Omega$ is the convex quadrilateral with vertices $(\underline{x}, \underline{x} / \bar{y}),(\bar{x}, \bar{x} / \bar{y})$, $(\bar{x}, \bar{x} / \underline{y})$, and $(\underline{x}, \underline{x} / \underline{y})$, there exist nonnegative scalars $\alpha_{1}, \alpha_{2}$, $\alpha_{3}$, and $\alpha_{4}$ such that $\alpha_{1}+\alpha_{2}+\alpha_{3}+\alpha_{4}=1$ and $\left(\tilde{x}, \tilde{z}_{1}\right)=$ $\alpha_{1}(\underline{x}, \underline{x} / \bar{y})+\alpha_{2}(\bar{x}, \bar{x} / \bar{y})+\alpha_{3}(\bar{x}, \bar{x} / \underline{y})+\alpha_{4}(\underline{x}, \underline{x} / \underline{y})$. Therefore,

$$
\begin{aligned}
\tilde{h}\left(\tilde{x}, \tilde{z}_{1}\right)= & \tilde{h}\left(\alpha_{1}(\underline{x}, \underline{x} / \bar{y})+\alpha_{2}(\bar{x}, \bar{x} / \bar{y})\right. \\
& \left.+\alpha_{3}(\bar{x}, \bar{x} / \underline{y})+\alpha_{4}(\underline{x}, \underline{x} / \underline{y})\right) \\
\geq & \alpha_{1} \tilde{h}(\underline{x}, \underline{x} / \bar{y})+\alpha_{2} \tilde{h}(\bar{x}, \bar{x} / \bar{y}) \\
& +\alpha_{3} \tilde{h}(\bar{x}, \bar{x} / \underline{y})+\alpha_{4} \tilde{h}(\underline{x}, \underline{x} / \underline{y}) \\
= & \alpha_{1} f(\underline{x}, \underline{x} / \bar{y})+\alpha_{2} f(\bar{x}, \bar{x} / \bar{y}) \\
& +\alpha_{3} f(\bar{x}, \bar{x} / \underline{y})+\alpha_{4} f(\underline{x}, \underline{x} / \underline{y}) \\
= & \alpha_{1} h(\underline{x}, \underline{x} / \bar{y})+\alpha_{2} h(\bar{x}, \bar{x} / \bar{y}) \\
& +\alpha_{3} h(\bar{x}, \bar{x} / \underline{y})+\alpha_{4} h(\underline{x}, \underline{x} / \underline{y}) \\
= & h\left(\alpha_{1}(\underline{x}, \underline{x} / \bar{y})+\alpha_{2}(\bar{x}, \bar{x} / \bar{y})\right. \\
& \left.+\alpha_{3}(\bar{x}, \bar{x} / \underline{y})+\alpha_{4}(\underline{x}, \underline{x} / \underline{y})\right) \\
= & h\left(\tilde{x}, \tilde{z}_{1}\right) .
\end{aligned}
$$

The inequality follows from the definition of a concave function; the two subsequent equalities from the fact that $h$ agrees with $f$ at the vertices of $\Omega$ and $f\left(x, z_{1}\right) \leq \tilde{h}\left(x, z_{1}\right) \leq h\left(x, z_{1}\right)$ for all $\left(x, z_{1}\right) \in \Omega$; and the last equality from the fact that $h$ is affine. The relation obtained $\tilde{h}\left(\tilde{x}, \tilde{z}_{1}\right) \geq h\left(\tilde{x}, \tilde{z}_{1}\right)$ contradicts $\tilde{h}\left(\tilde{x}, \tilde{z}_{1}\right)<h\left(\tilde{x}, \tilde{z}_{1}\right)$. So $h$ is the concave envelope of $f$ on $\Omega$.

A similar proof can be found in [30] on convex and concave envelopes of functions defined on convex quadrilaterals.

Proposition 2. The convex hull of $\mathcal{S}_{2}=\left\{\left(x, z_{1}, z_{2}\right) \in\right.$ $\left.\mathbb{R}^{3}: \underline{x} \leq x \leq \bar{x}, x / \bar{y} \leq z_{1} \leq x / \underline{y}, z_{2}=z_{1}^{2} / x\right\}$, where $0<\underline{x}<\bar{x}, 0<\underline{y}<\bar{y}$, is $\overline{\mathcal{S}}_{2}=\left\{\left(x, z_{1}, z_{2}\right) \in \mathbb{R}^{3}: \underline{x} \leq\right.$ $\left.x \leq \bar{x}, z_{1}^{2} \leq x z_{2}, x+\underline{y} \bar{y} z_{2} \leq(\underline{y}+\bar{y}) z_{1}\right\}$. tion 1.
Applying Proposition 2 to (5), we replace (7) in Model 1 by

$$
\mathrm{V}_{k k}+\underline{t}_{\ell} \bar{t}_{\ell} \mathrm{W}_{\ell \ell} \leq\left(\underline{t}_{\ell}+\bar{t}_{\ell}\right) \mathrm{W}_{k \ell} \forall \ell=(k, m) \in \mathcal{T},
$$

and define a new formulation of SDP relaxation in Model 3. We will refer to this relaxation as "semidefinite relaxation 2" (SDR2). The "tight-and-cheap relaxation 2" is given in Model 4. From Proposition 2, $\overline{\mathcal{S}}_{2} \subset \mathcal{S}_{3}$ and then SDR2 (respectively TCR2) is stronger than SDR1 (TCR1).
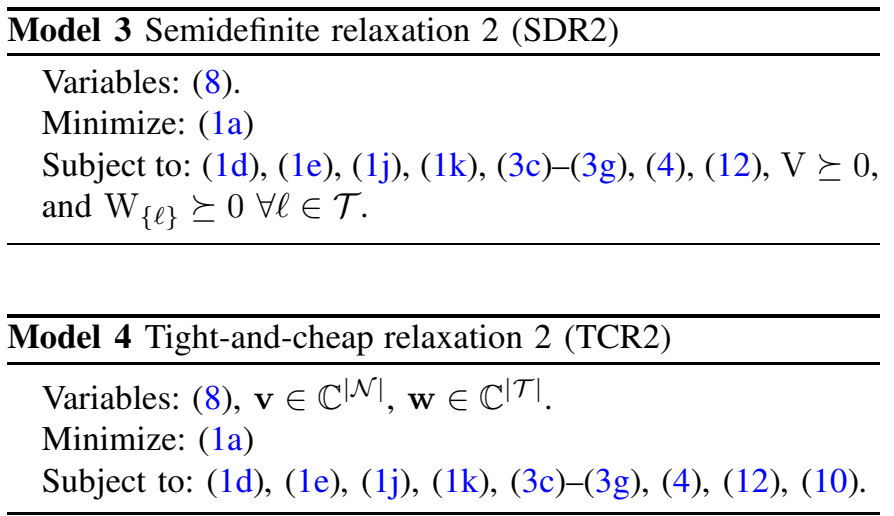

\section{ORPD: SOLUTION APPROACH}

Following [12], a mixed-integer nonlinear program (MINLP) is an optimization problem of the form

$$
\begin{aligned}
\hat{v}=\operatorname{minimize} & f_{0}(\boldsymbol{x}, \boldsymbol{y}) \\
\text { subject to } & f_{i}(\boldsymbol{x}, \boldsymbol{y}) \leq 0, \\
& \boldsymbol{x} \in \mathbb{R}^{n_{1}}, \boldsymbol{y} \in \mathbb{Z}^{n_{2}} .
\end{aligned}
$$

By fixing all integer variables, i.e. $\boldsymbol{y}=\tilde{\boldsymbol{y}} \in \mathbb{Z}^{n_{2}}$, we obtain the following subproblem (SP) of (15)

$$
\begin{aligned}
\hat{v}_{S P}=\operatorname{minimize} & f_{0}(\boldsymbol{x}, \tilde{\boldsymbol{y}}) \\
\text { subject to } & f_{i}(\boldsymbol{x}, \tilde{\boldsymbol{y}}) \leq 0, \quad i=1,2, \ldots, m, \\
& \boldsymbol{x} \in \mathbb{R}^{n_{1}} .
\end{aligned}
$$

Then we can rewrite the MINLP (15) as

$\hat{v}=\min _{\tilde{\boldsymbol{y}} \in \mathbb{Z}^{n_{2}}}\left\{\min _{\boldsymbol{x} \in \mathbb{R}^{n_{1}}}\left\{f_{0}(\boldsymbol{x}, \tilde{\boldsymbol{y}}): f_{i}(\boldsymbol{x}, \tilde{\boldsymbol{y}}) \leq 0, i=1,2, \ldots, m\right\}\right\}$

If (16) is feasible, its global optimal value $\hat{v}_{S P}$ provides an upper bound for $\hat{v}$ and the optimal solution $\left(\hat{\boldsymbol{x}}_{S P}, \tilde{\boldsymbol{y}}\right)$ of (16) is called a suboptimal solution of MINLP (15). The subproblem (16) may be nonconvex and solving it to global optimality may be hard. In this case, any local optimal value $\bar{v}_{S P}$ of (16) can be considered as an upper bound of $\hat{v}$.

Consider a relaxation (R) of (15) of the form

$$
\begin{aligned}
\hat{v}_{R}= & \operatorname{minimize} \underline{f}_{0}(\boldsymbol{x}, \boldsymbol{y}) \\
& \text { subject to }(\boldsymbol{x}, \boldsymbol{y}) \in \bar{\Omega} \subseteq \mathbb{R}^{n_{1}} \times \mathbb{R}^{n_{2}},
\end{aligned}
$$

where $\bar{\Omega}$ contains the feasible set of (15) and $\underline{f}_{0}$ underestimates $f_{0}$ for all feasible solutions of (15). The global optimal value $\hat{v}_{R}$ is a lower bound of $\hat{v}$. Since it is necessary to solve (17) to global optimality to obtain a lower bound on $\hat{v}$, it is more advantageous for the relaxation (17) to be convex. 
Let $\left(\hat{\boldsymbol{x}}_{R}, \hat{\boldsymbol{y}}_{R}\right)$ and $\hat{v}_{R}$ be respectively the global optimal solution and the global optimal value of a convex relaxation $(\mathrm{R})$ of a MINLP. Now, consider the subproblem (SP) associated to $\tilde{\boldsymbol{y}}_{R}$, the closest integer solution to $\hat{\boldsymbol{y}}_{R}$. We define the optimality gap of the relaxation (R) by $100\left(1-\hat{v}_{R} / \bar{v}_{S P}\right)$, where $\bar{v}_{S P}$ is a local optimal value of the subproblem (SP). If the optimality gap is close to zero, we say that the relaxation is tight and the suboptimal solution $\left(\hat{\boldsymbol{x}}_{S P}, \tilde{\boldsymbol{y}}_{R}\right)$ is near-global optimal for the MINLP.

This leads to the following approach to solve the ORPD problem (1):

1) Solve SDR1, TCR1, SDR2 or TCR2 and find corresponding shunt solutions $\hat{\boldsymbol{u}} \in[0,1]^{|\mathcal{U}|}$ and tap ratios $\hat{\boldsymbol{t}} \in \prod_{\ell \in \mathcal{T}}\left[\underline{t}_{\ell}, \bar{t}_{\ell}\right]$ with formulas (9),

2) Round-off $\boldsymbol{u}$ and $\boldsymbol{t}$ to their respective nearest discrete values $\tilde{\boldsymbol{u}} \in\{0,1\}^{|\mathcal{U}|}$ and $\tilde{\boldsymbol{t}} \in \prod_{\ell \in \mathcal{T}}\left\{\underline{t}_{\ell}, \ldots, \bar{t}_{\ell}\right\}$,

3) Fix $\boldsymbol{u}=\tilde{\boldsymbol{u}}$ and $\boldsymbol{t}=\tilde{\boldsymbol{t}}$ and solve the ACOPF subproblem with a nonlinear (local) solver.

\section{COMPUtATIONAL RESUlts}

In this section, we evaluate the accuracy and the computational efficiency of SDR1 and TCR1 as compared to SDR2 and TCR2.

In order to increase the computational speed of solving SDR1 and SDR2, we exploited the sparsity of a power network by replacing the SDP constraint $\mathrm{V} \succeq 0$ by small SDP constraints defined on a chordal extension of the power network. More details about exploiting the sparsity of power networks in SDP relaxations of the OPF problem can be found in [29], [31]-[34].

Let us interpret the network $\mathscr{P}=(\mathcal{N}, \mathcal{L})$ as a connected, simple and undirected graph $\mathscr{G}=(\mathcal{N}, \mathcal{E})$ where $\mathcal{N}=\{1, \ldots, n\}$ represents the set of vertices and $\mathcal{E}=$ $\{\{k, m\}:(k, m)$ or $(m, k) \in \mathcal{L}\}$, the set of edges. It was proved in [35] that the SDP constraint $\mathrm{V} \succeq 0$ in SDR1 or SDR2 is equivalent to $\mathrm{V}_{\mathcal{K}} \succeq 0$ for every maximal clique $\mathcal{K}$ of a chordal extension $\mathscr{G}^{\prime}$ of $\mathscr{G} . \mathrm{V}_{\mathcal{K}}$ is the submatrix of $\mathrm{V}$ in which the set of row indices that remain and the set of column indices that remain are both $\mathcal{K}$. To compute the maximal cliques of a chordal extension of $\mathscr{G}$, we used the same algorithm as in [29].

We tested the models 1, 2, 3, 4, on standard test cases available from MATPOWER [26], [36], [37]. We assigned a shunt variable $u_{k}$ to each shunt element connected to a bus $k \in \mathcal{U}$ and we assumed that the tap ratio $t_{\ell}$ of each transformer $\ell \in \mathcal{T}$ varies from 0.9 to $1.1 \mathrm{pu}$ by steps of $0.0125 \mathrm{pu}$. Table I lists the test cases along with the number of shunt elements $|\mathcal{U}|$ and the number of transformers $|\mathcal{T}|$.

We solved SDR1, TCR1, SDR2 and TCR2 in MATLAB using CVX 2.1 [38] with the solver MOSEK 8.0.0.60 and default precision (tolerance $\epsilon=1.49 \times 10^{-8}$ ). All relaxations were implemented as a MATLAB package, which is available on GitHub [39]. It requires that MATPOWER and CVX be installed and that the input instance be provided in MATPOWER format. MOSEK numerically failed to solve SDR1 for case3120sp.

All ACOPF subproblems were solved with the MATPOWER-solver MIPS. When MIPS numerically failed to solve subproblems for some test cases, marked with "*" in Table II and Table III, we then used the solver FMINCON. Among these subproblems, FMINCON did not converge to a feasible solution after 1000 iterations for case3012wp (SDR1), case3120sp (TCR1) in cost minimization, and case 3120 sp (SDR1, TCR1), case3375wp (SDR1, TCR1) in loss minimization.

All the computations were carried out on an Intel Core i7-6700 CPU a $3.40 \mathrm{GHz}$ computing platform.

We report results for two different objective functions: the generation cost $\sum_{g \in \mathcal{G}} c_{g 2} p_{G g}^{2}+c_{g 1} p_{G g}+c_{g 0}[\$ / \mathrm{h}]$ and the active power losses $\sum_{g \in \mathcal{G}} p_{G g}$ [MW]. Both objective functions of test cases from [37] are the same. We denote $\underline{v}$ the best lower bound which is the maximum value among $\hat{v}_{S D R 1}$, $\hat{v}_{S D R 2}, \hat{v}_{T C R 1}, \hat{v}_{T C R 2}$, respective optimal values of SDR1, TCR1, SDR2, and TCR2. The normalized $\hat{v}_{R}$ of a relaxation is measured as $\hat{v}_{R} / \underline{v} \leq 1$, where $\hat{v}_{R}$ is the relaxation's optimal value. Table II and Table III summarize the normalized optimal values of relaxations. The results support the following key points:

1) Among all relaxations, SDR2 is the strongest.

2) SDR1 (respectively SDR2) is stronger than TCR1 (respectively TCR2).

3) SDR1 and TCR2 are comparable.

For a relaxation, the local optimal value of the ACOPF subproblem obtained from the relaxation's optimal solution after rounding-off the discrete variables $\boldsymbol{u}$ and $\boldsymbol{t}$ is denoted $\bar{v}_{S P}$. The normalized $\bar{v}_{S P}$ in Table II and Table III is the value $\bar{v}_{S P} / \bar{v} \geq 1$, where $\bar{v}$ the best upper bound, i.e. the minimum value among all $\bar{v}_{S P}$. In Table II and Table III, we observe that:

1) In general, applying the round-off technique with SDR2 (respectively TCR2) provide tighter upper bounds than with SDR1 (respectively TCR1) for large-scale instances.

2) Applying the round-off technique with TCR2 is comparable to using SDR1.

Optimality gaps of the four relaxations are given in Table IV and Table $\mathrm{V}$. The optimality gap of a relaxation is measured as $100\left(1-\hat{v}_{R} / \bar{v}_{S P}\right)$, where $\hat{v}_{R}$ is the relaxation's optimal value and $\bar{v}_{S P}$ is a local optimal value of the corresponding subproblem. With the optimality gap, we can guarantee the near-global optimality of the suboptimal solution obtained with MIPS. Results in Table IV and Table V show that:

1) Suboptimal solutions obtained from SDR2's optimal solutions have the lowest guaranteed optimality gaps. We can say that they are near-global optimal for all but one test case: case_ACTIV_SG_500 (cost minimization).

2) In general, suboptimal solutions obtained from SDR1's and TCR2's optimal solutions have almost the same guaranteed optimality gaps.

3) Suboptimal solutions obtained from TCR1's optimal solutions have slightly larger optimality gaps.

The computation times required to solve SDR1, TCR1, SDR2, and TCR2 as reported by MOSEK are also shown in Table IV and Table V. We see that

1) Solving SDR1 (respectively TCR1) is as expensive as solving SDR2 (respectively TCR2) in general. 
TABLE I: Dimensions of test instances

\begin{tabular}{|c|c|c|c|c|}
\hline Test case & $|\mathcal{N}|$ & $|\mathcal{L}|$ & $|\mathcal{U}|$ & $|\mathcal{T}|$ \\
\hline \multicolumn{5}{|c|}{ Small-scale instances } \\
\hline case14 & 14 & 20 & 1 & \\
\hline case24_ieee_rts & 24 & 38 & 1 & \\
\hline case 30 & 30 & 41 & 2 & 0 \\
\hline case_ieee30 & 30 & 41 & 2 & 4 \\
\hline case39 & 39 & 46 & 0 & 12 \\
\hline case 57 & 57 & 80 & 3 & 17 \\
\hline case89pegase & 89 & 210 & 44 & 32 \\
\hline \multicolumn{5}{|c|}{ Medium-scale instances } \\
\hline case118 & 118 & 186 & 14 & \\
\hline case_ACTIV_SG_200 & 200 & 245 & 4 & 66 \\
\hline case_illinois200 & 200 & 245 & 4 & 66 \\
\hline case300 & 300 & 411 & 29 & 107 \\
\hline case_ACTIV_SG_500 & 500 & 597 & 15 & 131 \\
\hline \multicolumn{5}{|c|}{ Large-scale instances } \\
\hline case1354pegase & 1354 & 1991 & 1082 & 234 \\
\hline case2383wp & 2383 & 2896 & 0 & 164 \\
\hline case2736sp & 2736 & 3269 & 1 & 168 \\
\hline case2737sop & 2737 & 3269 & 5 & 169 \\
\hline case2746wop & 2746 & 3307 & 6 & 171 \\
\hline case2746wp & 2746 & 3279 & 0 & 171 \\
\hline case2869pegase & 2869 & 4582 & 2197 & 493 \\
\hline case3012wp & 3012 & 3572 & 9 & 201 \\
\hline case $3120 \mathrm{sp}$ & 3120 & 3693 & 9 & 206 \\
\hline case $3375 \mathrm{wp}$ & 3374 & 4161 & 9 & 381 \\
\hline
\end{tabular}

2) Solving SDR1 (respectively SDR2) is much more expensive than solving TCR1 (respectively TCR2) for largescale instances. The TCRs are on average 30 times faster than the SDRs.

Overall, we can see that TCR2 offers an interesting trade-off between the optimality gap and the computation time.

\section{CONCLUSION}

We proposed a tight SDP relaxation (SDR2) for the ORPD problem. This formulation is based on a standard SDP relaxation (SDR1) combined with a tight convex tap changer model. Experiments on selected MATPOWER instances with up to 3375 buses show that SDR2 is stronger than SDR1, and computationally comparable. From both SDR1 and SDR2, we derived tight-and-cheap relaxations TCR1 and TCR2, respectively.

A round-off technique based on a SDP relaxation of the ORPD problem instead of the nonconvex continuous relaxation was also proposed. Computational results show that applying the round-off technique with SDR2 and TCR2 provides suboptimal solutions which are near-global optimal. Both provide almost the same guaranteed optimality gaps, but TCR2 is computationally much less expensive for large-scale instances. In summary, the proposed TCR-based approach provides the best trade-off between optimality gap and computation time compared to the SDR-based approach.

\section{REFERENCES}

[1] J. Carpentier. Contribution to the economic dispatch problem. Bulletin de la Société française des électriciens, 3(8):431-447, 1962.

[2] Mary B. Cain, Richard P. ONeill, and Anya Castillo. History of optimal power flow and formulations. Federal Energy Regulatory Commission, pages $1-36,2012$.
[3] Stephen Frank, Ingrida Steponavice, and Steffen Rebennack. Optimal power flow: A bibliographic survey I. Energy Systems, 3(3):221-258, 2012.

[4] Stephen Frank, Ingrida Steponavice, and Steffen Rebennack. Optimal power flow: A bibliographic survey II. Energy Systems, 3(3):259-289, 2012.

[5] Florin Capitanescu. Critical review of recent advances and further developments needed in AC optimal power flow. Electric Power Systems Research, 136:57-68, 2016.

[6] Abhinav Verma. Power grid security analysis: An optimization approach. Columbia University, 2010.

[7] Karsten Lehmann, Alban Grastien, and Pascal Van Hentenryck. ACfeasibility on tree networks is NP-hard. IEEE Transactions on Power Systems, 31(1):798-801, 2016.

[8] Zhifang Yang, Haiwang Zhong, Qing Xia, and Chongqing Kang. Fundamental review of the OPF problem: challenges, solutions, and stateof-the-art algorithms. Journal of Energy Engineering, 144(1):04017075, 2017.

[9] Zhifang Yang, Anjan Bose, Haiwang Zhong, Ning Zhang, Qing Xia, and Chongqing Kang. Optimal reactive power dispatch with accurately modeled discrete control devices: A successive linear approximation approach. IEEE Transactions on Power Systems, 32(3):2435-2444, 2017.

[10] Pierre Bonami, Mustafa Kilinç, and Jeff Linderoth. Algorithms and software for convex mixed integer nonlinear programs. In Mixed integer nonlinear programming, pages 1-39. Springer, 2012.

[11] Jon Lee and Sven Leyffer. Mixed Integer Nonlinear Programming, volume 154. Springer Science \& Business Media, 2011.

[12] Samuel Burer and Adam N. Letchford. Non-convex mixed-integer nonlinear programming: A survey. Surveys in Operations Research and Management Science, 17(2):97-106, 2012.

[13] R. A. Jabr. Radial distribution load flow using conic programming. IEEE Transactions on Power Systems, 21(3):1458-1459, Aug 2006

[14] Xiaoqing Bai, Hua Wei, Katsuki Fujisawa, and Yong Wang. Semidefinite programming for optimal power flow problems. International Journal of Electrical Power \& Energy Systems, 30(6):383-392, 2008.

[15] Carleton Coffrin, Hassan L. Hijazi, and Pascal Van Hentenryck. The QC relaxation: A theoretical and computational study on optimal power flow. IEEE Transactions on Power Systems, 31(4):3008-3018, 2016.

[16] Hassan Hijazi, Carleton Coffrin, and Pascal Van Hentenryck. Convex quadratic relaxations for mixed-integer nonlinear programs in power systems. Mathematical Programming Computation, pages 1-47, 2014.

[17] Tao Ding, Shiyu Liu, Wei Yuan, Zhaohong Bie, and Bo Zeng. A twostage robust reactive power optimization considering uncertain wind power integration in active distribution networks. IEEE Transactions on Sustainable Energy, 7(1):301-311, 2016.

[18] Wenchuan Wu, Zhuang Tian, and Boming Zhang. An exact linearization method for OLTC of transformer in branch flow model. IEEE Transactions on Power Systems, 32(3):2475-2476, 2017.

[19] Burak Kocuk, Santanu S. Dey, and Xu Sun. New formulation and strong MISOCP relaxations for AC optimal transmission switching problem. IEEE Transactions on Power Systems, 32(6):4161-4170, 2017.

[20] Quentin Gemine, Damien Ernst, Quentin Louveaux, and Bertrand Cornélusse. Relaxations for multi-period optimal power flow problems with discrete decision variables. In 2014 Power Systems Computation Conference, pages 1-7. IEEE, 2014.

[21] Christian Bingane, Miguel F. Anjos, and Sébastien Le Digabel. CONICOPF: A tight-and-cheap conic relaxation with accuracy metrics for single-period and multi-period ACOPF problems. Technical Report G2019-19, Les cahiers du GERAD, 2019.

[22] Florin Capitanescu and Louis Wehenkel. Sensitivity-based approaches for handling discrete variables in optimal power flow computations. IEEE Transactions on Power Systems, 25(4):1780-1789, 2010.

[23] W.F. Tinney, J.M. Bright, K.D. Demaree, and B.A. Hughes. Some deficiencies in optimal power flow. IEEE Transactions on Power Systems, 3(2):676-683, 1988.

[24] Miguel F. Anjos and Jean B. Lasserre. Introduction to semidefinite, conic and polynomial optimization. In Handbook on Semidefinite, Conic and Polynomial Optimization, pages 1-22. Springer, 2012.

[25] Javad Lavaei. Zero duality gap for classical OPF problem convexifies fundamental nonlinear power problems. In Proceedings of the 2011 American Control Conference, pages 4566-4573. IEEE, 2011.

[26] R. D. Zimmerman, C. E. Murillo-Sanchez, and R. J. Thomas. MATPOWER: Steady-state operations, planning, and analysis tools for power systems research and education. IEEE Transactions on Power Systems, 26(1):12-19, Feb 2011. 
TABLE II: Cost minimization: Normalized optimal values

\begin{tabular}{|c|c|c|c|c|c|c|c|c|c|c|}
\hline \multirow[t]{2}{*}{ Test case } & \multirow[t]{2}{*}{$\underline{v}[\$ / \mathrm{h}]$} & \multirow[t]{2}{*}{$\bar{v}[\$ / \mathrm{h}]$} & \multicolumn{4}{|c|}{ Normalized $\hat{v}_{R}$} & \multicolumn{4}{|c|}{ Normalized $\bar{v}_{S P}$} \\
\hline & & & SDR1 & SDR2 & TCR1 & TCR2 & SDR1 & SDR2 & TCR1 & TCR2 \\
\hline \multicolumn{11}{|c|}{ Small-scale instances } \\
\hline case14 & 8078.62 & 8078.75 & 1.0000 & 1.0000 & 1.0000 & 1.0000 & 1.0000 & 1.0000 & 1.0000 & 1.0000 \\
\hline case24_ieee_rts & 63333.39 & 63335.67 & 1.0000 & 1.0000 & 0.9999 & 1.0000 & 1.0000 & 1.0000 & 1.0000 & 1.0000 \\
\hline $\operatorname{case} 30^{-}$ & 576.89 & 576.89 & 1.0000 & 1.0000 & 0.9993 & 0.9993 & 1.0000 & 1.0000 & 1.0000 & 1.0000 \\
\hline case_ieee 30 & 8902.67 & 8902.75 & 1.0000 & 1.0000 & 1.0000 & 1.0000 & 1.0000 & 1.0000 & 1.0000 & 1.0000 \\
\hline $\operatorname{case} 39$ & 41850.24 & 41852.45 & 1.0000 & 1.0000 & 1.0000 & 1.0000 & 1.0000 & 1.0000 & 1.0000 & 1.0000 \\
\hline case 57 & 41682.14 & 41688.61 & 0.9997 & 1.0000 & 0.9996 & 0.9999 & 1.0000 & 1.0000 & 1.0000 & 1.0000 \\
\hline case89pegase & 5803.58 & 5804.23 & 0.9996 & 1.0000 & 0.9992 & 0.9999 & 1.0001 & 1.0000 & 1.0001 & 1.0001 \\
\hline Average & & & 0.9998 & 1.0000 & 0.9996 & 0.9999 & 1.0000 & 1.0000 & 1.0000 & 1.0000 \\
\hline \multicolumn{11}{|c|}{ Medium-scale instances } \\
\hline case118 & 129526.00 & 129662.15 & 0.9987 & 1.0000 & 0.9987 & 0.9997 & 1.0003 & 1.0000 & 1.0003 & 1.0000 \\
\hline case_ACTIV_SG_200 & 27552.84 & 27553.52 & 1.0000 & 1.0000 & 1.0000 & 1.0000 & $* 1.0000$ & 1.0000 & $* 1.0000$ & 1.0000 \\
\hline case_illinois200 & 36738.07 & 36743.37 & 1.0000 & 1.0000 & 0.9998 & 0.9998 & $* 1.0000$ & 1.0000 & 1.0000 & 1.0000 \\
\hline case 300 & 718938.90 & 719154.24 & 0.9994 & 1.0000 & 0.9989 & 0.9992 & 1.0002 & 1.0000 & 1.0002 & 1.0002 \\
\hline case_ACTIV_SG_500 & 70316.47 & 72454.07 & 0.9875 & 1.0000 & 0.9751 & 0.9792 & 1.0016 & 1.0033 & 1.0000 & 1.0018 \\
\hline Average & & & 0.9950 & 1.0000 & 0.9902 & 0.9919 & 1.0007 & 1.0013 & 1.0001 & 1.0007 \\
\hline \multicolumn{11}{|c|}{ Large-scale instances } \\
\hline case1354pegase & 73999.72 & 74005.77 & 0.9998 & 1.0000 & 0.9995 & 0.9998 & 1.0000 & 1.0000 & 1.0000 & 1.0000 \\
\hline case2383wp & 1856849.35 & 1862626.70 & 0.9996 & 1.0000 & 0.9981 & 0.9992 & 1.0039 & 1.0000 & 1.0068 & 1.0033 \\
\hline case2736sp & 1306771.84 & 1307134.47 & 0.9997 & 1.0000 & 0.9988 & 0.9998 & 1.0001 & 1.0000 & 1.0002 & 1.0001 \\
\hline case2737sop & 777020.01 & 777337.82 & 0.9997 & 1.0000 & 0.9990 & 0.9997 & 1.0001 & 1.0000 & 1.0001 & 1.0001 \\
\hline case 2746 wop & 1206874.60 & 1207872.71 & 0.9994 & 1.0000 & 0.9983 & 0.9994 & 1.0001 & 1.0000 & 1.0002 & 1.0001 \\
\hline case2746wp & 1630617.28 & 1631018.94 & 0.9997 & 1.0000 & 0.9988 & 0.9996 & 1.0001 & 1.0000 & 1.0002 & 1.0000 \\
\hline case2869pegase & 133867.21 & 133877.91 & 0.9998 & 1.0000 & 0.9994 & 0.9997 & 1.0000 & 1.0000 & 1.0000 & 1.0000 \\
\hline case3012wp & 2578533.79 & 2590254.80 & 0.9987 & 1.0000 & 0.9968 & 0.9985 & - & 1.0000 & 1.0009 & 1.0013 \\
\hline case $3120 \mathrm{sp}$ & 2135933.93 & 2143654.67 & - & 1.0000 & 0.9975 & 0.9988 & - & 1.0002 & - & 1.0000 \\
\hline case $3375 \mathrm{wp}$ & 7397491.46 & *7 409223.47 & 0.9998 & 1.0000 & 0.9988 & 0.9994 & $* 1.0002$ & $* 1.0000$ & $* 1.0002$ & $* 1.0004$ \\
\hline Average & & & - & 1.0000 & 0.9985 & 0.9994 & - & 1.0000 & - & 1.0005 \\
\hline
\end{tabular}

TABLE III: Loss minimization: Normalized optimal values

\begin{tabular}{|c|c|c|c|c|c|c|c|c|c|c|}
\hline \multirow[t]{2}{*}{ Test case } & \multirow[t]{2}{*}{$\underline{v}[\mathrm{MW}]$} & \multirow[t]{2}{*}{$\bar{v}[\mathrm{MW}]$} & \multicolumn{4}{|c|}{ Normalized $\hat{v}_{R}$} & \multicolumn{4}{|c|}{ Normalized $\bar{v}_{S P}$} \\
\hline & & & SDR1 & SDR2 & TCR1 & TCR2 & SDR1 & SDR2 & TCR1 & TCR2 \\
\hline \multicolumn{11}{|c|}{ Small-scale instances } \\
\hline case14 & 259.49 & 259.49 & 1.0000 & 1.0000 & 1.0000 & 1.0000 & 1.0000 & 1.0000 & 1.0000 & 1.0000 \\
\hline case24_ieee_rts & 2875.33 & 2875.37 & 1.0000 & 1.0000 & 0.9999 & 1.0000 & 1.0000 & 1.0000 & 1.0000 & 1.0000 \\
\hline case30 & 191.09 & 191.09 & 1.0000 & 1.0000 & 0.9999 & 0.9999 & 1.0000 & 1.0000 & 1.0000 & 1.0000 \\
\hline case_ieee30 & 284.68 & 284.71 & 0.9997 & 1.0000 & 0.9997 & 1.0000 & 1.0000 & 1.0000 & 1.0000 & 1.0000 \\
\hline case39 & 6283.20 & 6283.44 & 1.0000 & 1.0000 & 1.0000 & 1.0000 & 1.0000 & 1.0000 & 1.0000 & 1.0000 \\
\hline case 57 & 1260.83 & 1260.98 & 0.9998 & 1.0000 & 0.9998 & 1.0000 & 1.0000 & 1.0000 & 1.0000 & 1.0000 \\
\hline case89pegase & 5803.58 & 5804.23 & 0.9996 & 1.0000 & 0.9992 & 0.9999 & 1.0001 & 1.0000 & 1.0001 & 1.0001 \\
\hline Average & & & 0.9998 & 1.0000 & 0.9997 & 1.0000 & 1.0000 & 1.0000 & 1.0000 & 1.0000 \\
\hline \multicolumn{11}{|c|}{ Medium-scale instances } \\
\hline case118 & 4250.75 & 4251.17 & 1.0000 & 1.0000 & 1.0000 & 1.0000 & 1.0000 & 1.0000 & 1.0000 & 1.0000 \\
\hline case_ACTIV_SG_200 & 1483.23 & 1483.32 & 1.0000 & 1.0000 & 0.9999 & 0.9999 & 1.0000 & 1.0000 & $* 1.0000$ & 1.0000 \\
\hline case_illinois200 & 2245.96 & 2246.22 & 1.0000 & 1.0000 & 0.9999 & 0.9999 & $* 1.0000$ & 1.0000 & 1.0000 & 1.0000 \\
\hline case $\overline{3} 00$ & 23723.13 & 23725.42 & 0.9999 & 1.0000 & 0.9996 & 0.9997 & 1.0000 & 1.0001 & 1.0001 & 1.0001 \\
\hline case_ACTIV_SG_500 & 7815.64 & 7817.25 & 1.0000 & 1.0000 & 0.9998 & 0.9998 & 1.0000 & 1.0000 & 1.0000 & 1.0000 \\
\hline Average & & & 1.0000 & 1.0000 & 0.9998 & 0.9999 & 1.0000 & 1.0000 & 1.0000 & 1.0000 \\
\hline \multicolumn{11}{|c|}{ Large-scale instances } \\
\hline case1354pegase & 73999.72 & 74005.77 & 0.9998 & 1.0000 & 0.9995 & 0.9998 & 1.0000 & 1.0000 & 1.0000 & 1.0000 \\
\hline case2383wp & 24967.98 & 24983.29 & 1.0000 & 1.0000 & 0.9992 & 0.9997 & 1.0000 & 1.0000 & 1.0001 & 1.0001 \\
\hline case2736sp & 18324.27 & 18329.28 & 0.9999 & 1.0000 & 0.9993 & 0.9998 & 1.0001 & 1.0000 & 1.0001 & 1.0000 \\
\hline case2737sop & 11391.09 & 11394.38 & 0.9997 & 1.0000 & 0.9992 & 0.9997 & 1.0001 & 1.0000 & 1.0001 & 1.0001 \\
\hline case 2746 wop & 19197.71 & 19209.58 & 0.9996 & 1.0000 & 0.9989 & 0.9996 & 1.0001 & 1.0000 & 1.0001 & 1.0001 \\
\hline case2746wp & 25255.49 & 25260.72 & 0.9999 & 1.0000 & 0.9992 & 0.9997 & 1.0001 & 1.0000 & 1.0001 & 1.0000 \\
\hline case2869pegase & 133867.21 & 133877.91 & 0.9998 & 1.0000 & 0.9994 & 0.9997 & 1.0000 & 1.0000 & 1.0000 & 1.0000 \\
\hline case3012wp & 27611.41 & 27648.22 & 0.9995 & 1.0000 & 0.9986 & 0.9994 & 1.0001 & 1.0000 & 1.0001 & 1.0003 \\
\hline case3120sp & 21463.22 & 21524.52 & 0.9998 & 1.0000 & 0.9991 & 0.9997 & - & 1.0000 & - & 1.0000 \\
\hline case3375wp & 48950.55 & 49002.59 & 0.9997 & 1.0000 & 0.9993 & 0.9995 & - & 1.0027 & - & 1.0000 \\
\hline Average & & & 0.9998 & 1.0000 & 0.9992 & 0.9996 & - & 1.0003 & - & 1.0001 \\
\hline
\end{tabular}


TABLE IV: Cost minimization: Optimality gaps and computation times

\begin{tabular}{|c|c|c|c|c|c|c|c|c|}
\hline \multirow[t]{2}{*}{ Test case } & \multicolumn{4}{|c|}{ Optimality gap [\%] } & \multicolumn{4}{|c|}{ Computation time $[\mathrm{s}]$} \\
\hline & SDR1 & SDR2 & TCR1 & TCR2 & SDR1 & SDR2 & TCR1 & TCR2 \\
\hline \multicolumn{9}{|c|}{ Small-scale instances } \\
\hline case14 & 0.00 & 0.00 & 0.00 & 0.00 & 0.15 & 0.13 & 0.12 & 0.36 \\
\hline case24_ieee_rts & 0.01 & 0.00 & 0.01 & 0.00 & 0.19 & 0.55 & 0.54 & 0.28 \\
\hline case 30 & 0.00 & 0.00 & 0.07 & 0.07 & 0.12 & 0.12 & 0.20 & 0.20 \\
\hline case_ieee 30 & 0.00 & 0.00 & 0.00 & 0.00 & 0.12 & 0.11 & 0.13 & 0.14 \\
\hline case39 & 0.01 & 0.01 & 0.01 & 0.01 & 0.43 & 0.41 & 0.61 & 0.52 \\
\hline case 57 & 0.05 & 0.02 & 0.05 & 0.03 & 0.47 & 0.21 & 0.20 & 0.24 \\
\hline case89pegase & 0.06 & 0.01 & 0.10 & 0.03 & 2.26 & 1.64 & 0.76 & 0.72 \\
\hline Average & 0.03 & 0.01 & 0.05 & 0.02 & 0.91 & 0.69 & 0.45 & 0.42 \\
\hline \multicolumn{9}{|c|}{ Medium-scale instances } \\
\hline case118 & 0.27 & 0.11 & 0.27 & 0.13 & 0.30 & 0.36 & 0.38 & 0.40 \\
\hline case_ACTIV_SG_200 & $* 0.00$ & 0.00 & $* 0.01$ & 0.01 & 0.82 & 0.79 & 0.57 & 0.55 \\
\hline case_illinois200 & $* 0.02$ & 0.01 & 0.03 & 0.03 & 0.94 & 1.41 & 0.73 & 0.75 \\
\hline case 300 & 0.11 & 0.03 & 0.16 & 0.13 & 1.23 & 2.25 & 0.99 & 0.89 \\
\hline case_ACTIV_SG_500 & 4.31 & 3.27 & 5.36 & 5.14 & 4.37 & 4.53 & 3.85 & 3.14 \\
\hline Average & 1.69 & 1.26 & 2.10 & 2.00 & 2.23 & 2.60 & 1.92 & 1.63 \\
\hline \multicolumn{9}{|c|}{ Large-scale instances } \\
\hline case1354pegase & 0.03 & 0.01 & 0.06 & 0.03 & 13.35 & 11.95 & 7.53 & 6.66 \\
\hline case2383wp & 0.74 & 0.31 & 1.17 & 0.71 & 307.06 & 325.67 & 14.19 & 12.97 \\
\hline case $2736 \mathrm{sp}$ & 0.07 & 0.03 & 0.16 & 0.07 & 426.85 & 398.85 & 13.84 & 12.05 \\
\hline case2737sop & 0.08 & 0.04 & 0.16 & 0.08 & 381.34 & 372.61 & 11.23 & 12.75 \\
\hline case 2746 wop & 0.15 & 0.08 & 0.27 & 0.15 & 477.80 & 425.23 & 10.96 & 11.68 \\
\hline case2746wp & 0.06 & 0.02 & 0.16 & 0.07 & 418.71 & 414.08 & 11.27 & 11.19 \\
\hline case2869pegase & 0.03 & 0.01 & 0.07 & 0.04 & 55.71 & 80.07 & 21.27 & 19.78 \\
\hline case3012wp & - & 0.45 & 0.87 & 0.73 & 1670.60 & 1618.99 & 12.27 & 12.17 \\
\hline case3120sp & - & 0.38 & - & 0.48 & 763.19 & 672.87 & 13.64 & 13.22 \\
\hline case3375wp & $* 0.20$ & $* 0.16$ & $* 0.29$ & ${ }^{*} 0.24$ & 1638.91 & 1763.90 & 20.47 & 18.89 \\
\hline Average & - & 0.16 & - & 0.27 & 684.22 & 678.29 & 14.15 & 13.63 \\
\hline
\end{tabular}

TABLE V: Loss minimization: Optimality gaps and computation times

\begin{tabular}{|c|c|c|c|c|c|c|c|c|}
\hline \multirow[t]{2}{*}{ Test case } & \multicolumn{4}{|c|}{ Optimality gap [\%] } & \multicolumn{4}{|c|}{ Computation time [s] } \\
\hline & SDR1 & SDR2 & TCR1 & TCR2 & SDR1 & SDR2 & TCR1 & TCR2 \\
\hline \multicolumn{9}{|c|}{ Small-scale instances } \\
\hline case14 & 0.00 & 0.00 & 0.00 & 0.00 & 0.12 & 0.12 & 0.12 & 0.12 \\
\hline case24_ieee_rts & 0.00 & 0.00 & 0.00 & 0.00 & 0.16 & 0.18 & 0.39 & 0.20 \\
\hline case 30 & 0.00 & 0.00 & 0.01 & 0.01 & 0.13 & 0.13 & 0.17 & 0.17 \\
\hline case_ieee 30 & 0.04 & 0.01 & 0.05 & 0.02 & 0.16 & 0.34 & 0.49 & 0.28 \\
\hline case 39 & 0.00 & 0.00 & 0.00 & 0.00 & 0.17 & 0.19 & 0.22 & 0.22 \\
\hline case 57 & 0.03 & 0.01 & 0.03 & 0.01 & 0.19 & 0.23 & 0.18 & 0.49 \\
\hline case89pegase & 0.06 & 0.01 & 0.10 & 0.03 & 2.26 & 1.64 & 0.76 & 0.72 \\
\hline Average & 0.03 & 0.01 & 0.04 & 0.02 & 0.82 & 0.66 & 0.41 & 0.43 \\
\hline \multicolumn{9}{|c|}{ Medium-scale instances } \\
\hline case118 & 0.01 & 0.01 & 0.01 & 0.01 & 0.29 & 0.27 & 0.30 & 0.30 \\
\hline case_ACTIV_SG_200 & 0.01 & 0.01 & $* 0.01$ & 0.01 & 0.73 & 0.74 & 0.48 & 1.06 \\
\hline case_illinois 200 & $* 0.01$ & 0.01 & 0.02 & 0.02 & 1.83 & 0.92 & 1.70 & 0.74 \\
\hline case 300 & 0.02 & 0.02 & 0.06 & 0.05 & 1.02 & 0.91 & 0.98 & 0.88 \\
\hline case_ACTIV_SG_500 & 0.03 & 0.02 & 0.04 & 0.04 & 5.82 & 4.56 & 4.59 & 3.95 \\
\hline Average & 0.02 & 0.02 & 0.04 & 0.03 & 2.85 & 2.21 & 2.32 & 2.00 \\
\hline \multicolumn{9}{|c|}{ Large-scale instances } \\
\hline case1354pegase & 0.03 & 0.01 & 0.06 & 0.03 & 13.35 & 11.95 & 7.53 & 6.66 \\
\hline case2383wp & 0.07 & 0.06 & 0.15 & 0.10 & 252.55 & 271.06 & 13.94 & 12.56 \\
\hline case $2736 \mathrm{sp}$ & 0.05 & 0.03 & 0.11 & 0.05 & 434.61 & 280.45 & 10.52 & 9.58 \\
\hline case2737sop & 0.06 & 0.03 & 0.12 & 0.06 & 210.99 & 215.05 & 8.67 & 8.55 \\
\hline case 2746 wop & 0.10 & 0.06 & 0.18 & 0.12 & 394.81 & 444.52 & 9.88 & 11.20 \\
\hline case 2746 wp & 0.04 & 0.02 & 0.11 & 0.05 & 431.06 & 403.43 & 9.70 & 9.72 \\
\hline case2869pegase & 0.03 & 0.01 & 0.07 & 0.04 & 55.71 & 80.07 & 21.27 & 19.78 \\
\hline case3012wp & 0.19 & 0.13 & 0.28 & 0.22 & 2354.15 & 2426.65 & 16.82 & 17.45 \\
\hline case3120sp & - & 0.28 & - & 0.32 & 1193.18 & 973.71 & 16.66 & 19.16 \\
\hline case3375wp & - & 0.38 & - & 0.15 & 1415.74 & 1588.19 & 28.25 & 39.33 \\
\hline Average & - & 0.12 & - & 0.12 & 753.59 & 749.07 & 15.09 & 16.54 \\
\hline
\end{tabular}

[27] Xinda Ke, Nader Samaan, Jesse Holzer, Renke Huang, Bharat Vyakaranam, Mallikarjuna Vallem, Marcelo Elizondo, Ning Lu, Xiangqi Zhu, Brant Werts, et al. Coordinative real-time sub-transmission voltvar control for reactive power regulation between transmission and distribution systems. IET Generation, Transmission \& Distribution, 2018.

[28] Brett A. Robbins, Hao Zhu, and Alejandro D. Domínguez-García. Optimal tap setting of voltage regulation transformers in unbalanced distribution systems. IEEE Transactions on Power Systems, 31(1):256267, 2016.

[29] Christian Bingane, Miguel F. Anjos, and Sébastien Le Digabel. Tightand-cheap conic relaxation for the AC optimal power flow problem. IEEE Transactions on Power Systems, 33(6):7181-7188, 2018.

[30] Harold P. Benson. On the construction of convex and concave envelope formulas for bilinear and fractional functions on quadrilaterals. Computational Optimization and Applications, 27(1):5-22, 2004.

[31] R. A. Jabr. Exploiting sparsity in SDP relaxations of the OPF problem. IEEE Transactions on Power Systems, 27(2):1138-1139, May 2012.
[32] S. H. Low. Convex relaxation of optimal power flow-Part I: Formulations and equivalence. IEEE Transactions on Control of Network Systems, 1(1):15-27, March 2014.

[33] Martin S. Andersen, Anders Hansson, and Lieven Vandenberghe. Reduced-complexity semidefinite relaxations of optimal power flow problems. IEEE Transactions on Power Systems, 29(4):1855-1863, 2014.

[34] R. Madani, M. Ashraphijuo, and J. Lavaei. OPF solver. https://lavaei.ieor.berkeley.edu/Software.html, 2014.

[35] Robert Grone, Charles R. Johnson, Eduardo M. Sá, and Henry Wolkowicz. Positive definite completions of partial Hermitian matrices. Linear algebra and its applications, 58:109-124, 1984.

[36] A. B. Birchfield, T. Xu, K. M. Gegner, K. S. Shetye, and T. J. Overbye. Grid structural characteristics as validation criteria for synthetic networks. IEEE Transactions on Power Systems, 32(4):3258-3265, July 2017.

[37] Cédric Josz, Stéphane Fliscounakis, Jean Maeght, and Patrick Panciatici. AC power flow data in MATPOWER and QCQP format: iTesla, RTE snapshots, and PEGASE. arXiv preprint arXiv:1603.01533, 2016.

[38] CVX Research, Inc. CVX: MATLAB software for disciplined convex programming, version 2.1. http://cvxr.com/cvx, August 2012.

[39] Christian Bingane, Miguel F. Anjos, and Sébastien Le Digabel. CONICOPF: Conic relaxations of the optimal power flow problem. https://github.com/cbingane/conicopf, March 2019.

Christian Bingane (S'18) received the B.Eng. degree in electrical engineering in 2014 from Polytechnique Montreal, Montreal, QC, Canada, where he is currently working toward the Ph.D. degree in applied mathematics. He is currently a student member of the GERAD research center.

His research interests include optimization in power systems and conic programming. He is concerned with using linear programming, second-order cone programming or semidefinite programming to provide guaranteed global optimal solution to the optimal power flow problem for a large-scale power system.

Miguel F. Anjos (M'07-SM'18) received the B.Sc., the M.S. and the Ph.D. degrees from McGill University, Montreal, QC, Canada; Stanford University, Stanford, CA, USA; and the University of Waterloo, Waterloo, ON, Canada in 1992, 1994 and 2001 respectively.

He is currently Chair of Operational Research at the School of Mathematics, University of Edinburgh, Edinburgh, Scotland, UK, and Professor with the Department of Mathematics and Industrial Engineering, Polytechnique Montreal, Montreal, QC, Canada, where he holds the NSERC-Hydro-QuebecSchneider Electric Industrial Research Chair, and an Inria International Chair. $\mathrm{He}$ is a Licensed Professional Engineer in Ontario, Canada. He served for five years as Editor-in-Chief of Optimization and Engineering, and serves on several editorial boards.

His allocades include a Canada Research Chair, the Méritas Teaching Award, a Humboldt Research Fellowship, the title of EUROPT Fellow, and the Queen Elizabeth II Diamond Jubilee Medal. He is an elected Fellow of the Canadian Academy of Engineering.

Sébastien Le Digabel received the M.Sc.A. and the Ph.D. degrees in applied mathematics from Polytechnique Montreal, Montreal, Quebec, Canada in 2002 and 2008 respectively. He was a postdoctoral fellow with the IBM Watson Research Center and the University of Chicago in 2010 and 2011.

$\mathrm{He}$ is currently an Associate Professor with the Department of Mathematics and Industrial Engineering, Polytechnique Montreal, Montreal, QC, Canada, and a regular member of the GERAD research center.

His research interests include the analysis and development of algorithms for derivative-free and blackbox optimization, and the design of related software. All of his work on derivative-free optimization is included in the NOMAD software, a free package for blackbox optimization available at www.gerad.ca/nomad. 